\title{
TRATAMIENTO FARMACOLÓGICO DE LA DEPRESIÓN EN CÁNCER
}

\author{
PHARMACOLOGICAL TREATMENT OF DEPRESSION IN PATIENTS WITH CANCER
}

\author{
Patricia Bernal García' y María José Muñoz Algar² \\ ' Hospital Dr. Rodríguez Lafora. Carretera de Colmenar, Km 13,800. Madrid, España. \\ 2 Instituto Psiquiátrico Montreal. El Plantío, Majadahonda. Madrid, España.
}

Resumen

Objetivo: La depresión mayor es uno de los trastornos psiquiátricos más ampliamente reconocidos en los enfermos de cáncer. En España alrededor de un $\mathbf{1 6 , 4 9 \%}$ de los pacientes oncológicos desarrollan un Trastorno Depresivo Mayor. Cuando la sintomatología depresiva interfiere en el adecuado funcionamiento del paciente oncológico, los antidepresivos son el tratamiento de primera línea. El objetivo de este estudio es analizar la eficacia de las distintas opciones farmacológicas utilizadas para la depresión en pacientes oncológicos.

Método: Se realiza una búsqueda bibliográfica en la base de datos PubMed mediante los términos: <cancer AND depression treatment AND antidepressant $>$. Se analizaron un total de 36 artículos con un diseño tipo ensayo clínico.

Resultados: Se ha encontrado que los fármacos inhibidores de la recaptación de serotonina (ISRS) son eficaces para el tratamiento de la depresión en pacientes con cáncer. La paroxetina es una de las más eficaces. Los antidepresivos de perfil dual también han demostrado eficacia por su rapidez de acción y su utilidad en síntomas colaterales como los síntomas vasomotores y los dolores. La mirtazapina es de gran utilidad en síntomas como el dolor, náuseas, insomnio y ansiedad.

Conclusiones: La elección de la medicación debe estar basada en el perfil de síntomas del paciente, el potencial beneficio, la tolerabili-

\section{Abstract}

Objective: Major depression is one of the most prevalent diagnosis in oncological patients. In Spain, about $16-49 \%$ of patients develop a major depressive disorder. When the depressive symptoms interfere with the oncological patient's functionality, the antidepressant are the first line treatment. The objective of the present study is to analyze the effectiveness of different pharmacological options used for depression in oncological patients.

Method: We performed a bibliographic review in pubmed database with the terms: <cancer AND depression treatment AND antidepressant $>$. We analyzed 36 scientific articles with a clinical trial design.

Results: we have found the inhibitors selective serotonin reuptakes (ISSR) are effective in treating depression in cancer patient. paroxetine is one of the most effective. The dual antidepressants also have shown efficacy for its speed of action and their utility in side symptoms like vasomotor symptoms and pain. Mirtazapine is useful to treat pain, sickness, insomnia and anxiety.

Conclusion: the election of medication must be based on the symptoms profile of patient, the potential benefit, tolerability and risk/benefits with the purpose of design a strategy for each patient. The drugs most effective and tolerable in our study have

\section{Correspondencia:}


dad y el riesgo/beneficio con el propósito de diseñar una estrategia para cada paciente. Los fármacos que han demostrado mayor eficacia y tolerabilidad en nuestro estudio han sido los ISRS, la mirtazapina y los antidepresivos de acción dual.

Palabras clave: Depresión, tratamiento, cáncer, antidepresivos, serotonina. been the ISSR, the mirtazapine and the dual action antidepressants.

Keywords: Depression, treatment, cancer, antidepressant, serotonin.

\section{INTRODUCCIÓN}

El trastorno psiquiátrico que se asocia con más frecuencia al cáncer es la depresión ${ }^{(1)}$. No solo produce sufrimiento, sino también una peor calidad de vida. Se ha visto que reduce el cumplimiento del tratamiento anticancerígeno, puede Ilevar al suicidio, es una carga psicológica para la familia y prolonga el tiempo de hospitalización ${ }^{(2)}$. No siempre es fácil detectarla, por lo que es habitualmente infradiagnosticado $^{(3)}$. La terapia óptima para la depresión en pacientes con cáncer es incierta y hay evidencia limitada que apoye el uso de farmacoterapia ${ }^{(4)}$.

\section{Prevalencia}

Las estimaciones sobre prevalencia de la depresión en enfermos de cáncer varían desde el $1-50 \%{ }^{(5)}$. Esta variación es debida a la falta de estandarización en género y edad de la población evaluada, localización y etapa de la enfermedad, tamaño muestral, puntos de corte, instrumentos de evaluación, tipos de medida y criterios diagnósticos utilizados ${ }^{(6)}$.

El National Cancer Institute (NCl, 2016) estima que la depresión afecta a una proporción de entre un $15 \%$ a un $25 \%$ de los pacientes con esta enfermedad. En un alto porcentaje aparecen síntomas depresivos en las primeras semanas tras el diagnostico inicial. Sin embargo, la mayor parte acabará desarrollando un trastorno depresivo mayor ${ }^{(7)}$.
En España, se ha encontrado que alrededor de un 16-49\% de los pacientes oncológicos desarrollan un Trastorno Depresivo Mayor ${ }^{(8)}$.

\section{Diagnóstico}

El diagnostico de depresión mayor en pacientes de cáncer ha sido un desafío porque los criterios establecidos en DSM-IV incluyen un número de síntomas somáticos que han sido frecuentemente atribuidos al cáncer (pérdida de apetito, pérdida de peso, insomnio, fatiga, energía y disminución de la capacidad de concentración o razonamiento) o a los tratamientos utilizados como la quimioterapia y la radioterapia. Es por esta razón que está infradiagnosticado ${ }^{(9)}$.

Pero es muy importante este diagnóstico porque además del sufrimiento personal que acarrea, también afecta a la evolución de la enfermedad. El estilo de afrontamiento depresivo se relaciona con un menor tiempo de supervivencia ${ }^{(10)}$. Además alarga el tiempo de hospitalización, empeora la adherencia terapéutica y disminuye la calidad de vida ${ }^{(11)}$.

La depresión puede tener comorbilidad con otros trastornos mentales (de ansiedad y de personalidad) y con patologías médicas como las oncológicas.

Algunos autores $^{(12)}$ señalan una serie de síntomas que deben estimarse para el diagnóstico diferencial del trastorno depresivo reactivo a patología médica como son: anhedonia, tristeza, pérdida de tono, enlentecimiento psicomotor, antecedentes familiares 
de depresión, despertar temprano y mejoría vespertina del ánimo, mejoría con el ejercicio físico de síntomas depresivos, fatiga, anorexia, pérdida de peso y dolor. Aunque los criterios originales del DSM-IV (Manual Estadístico y Diagnóstico de la Asociación Americana de Psicología) excluyen síntomas que claramente son atribuidos a la condición médica, el diagnóstico diferencial es a menudo difícil o prácticamente imposible ${ }^{(2)}$.

Sería aconsejable realizar una entrevista en el ámbito de la atención primaria para detectar signos/síntomas que nos ayuden a realizar un diagnóstico diferencial. Se podría recabar información sobre fármacos que pudieran estar causando esta sintomatología, determinar otros trastornos psiquiátricos y descartar otras causas, así como una valoración del estado médico para detectar patologías asociadas o causantes de la depresión. Pero en este nivel de atención sanitaria hay dificultades para evaluar en todos los posibles pacientes este riesgo de patología afectiva.

Por otro lado, se podrían realizar otros procedimientos de cribaje para ahorrar el tiempo y los costes que derivarían de realizar de forma universal entrevistas diagnósticas. Con esto se conseguiría una detección temprana de personas en riesgo susceptibles de recibir una intervención psicológica de forma preferente ${ }^{(13)}$.

A pesar de esta dificultad existente en el diagnóstico se cuenta con diversos instrumentos para la evaluación psicológica:

- Escala de Ansiedad y Depresión Hospitalaria (HADS). Está validada en todos los idiomas y tipos de enfermedades.

- Cuestionario de Salud General de Goldberg (GHQ-28). Detecta con alta especificidad y sensibilidad pacientes oncológicos que presentan síntomas psiquiátricos.

- Screening psicosocial para el Cáncer (PPSCAN). Se evalúan 6 dimen- siones relevantes en la depresión, mediante 21 ítems. Estas son: salud en relación a calidad de vida, ansiedad, ánimo deprimido, apoyo social deseado y el percibido ${ }^{(14)}$.

Cuando a un paciente se le identifica con riesgo para el trastorno depresivo se debe realizar una entrevista clínica, considerando los criterios diagnósticos. Los más frecuentemente utilizados en enfermos con cáncer son: RCD (Criterios Diagnósticos de Investigación), El criterio Endicott y el DSM-IV ${ }^{(7)}$.

\section{Tratamiento de la depresión}

Los tratamientos de la depresión en pacientes oncológicos pueden dividirse en dos tipos:

- Biológicos

a) Psicofarmacológico.

b) Terapia electroconvulsiva. Se considera como último recurso en pacientes que precisan de hospitalización por ser resistentes al tratamiento antidepresivo o por la severidad del síndrome depresivo. Se induce un ataque de gran mal que libera noradrenalina y dopamina y esto mejora notablemente el funcionamiento del sistema de aminas. Es un tratamiento de primera línea cuando el riesgo de la medicación antidepresiva es superior al de la terapia electroconvulsiva, cuando se precisa de una respuesta rápida al tratamiento, cuando hay historia previa de repuesta positiva a este tratamiento o historia previa de mala respuesta a los antidepresivos. Si la depresión se acompaña de síntomas psicóticos o de estupor o marcado retardo psicomotor es especialmente efectiva ${ }^{(15)}$. 
- Psicológicos

a) Psicoterapia individual

b) Psicoterapia de grupo

c) Grupos de autoayuda

El objetivo de las intervenciones psicoterapéuticas durante el tratamiento de pacientes oncológicos es reducir dificultades y conseguir la estabilidad psíquica. Un reciente meta-análisis sobre la eficacia del tratamiento psicológico y farmacológico en pacientes con cáncer y marcados síntomas depresivos, ha demostrado que los dos abordajes son efectivos y que el tratamiento integrado podría obtener aún mejores resultados ${ }^{(16)}$.

La ausencia de tratamiento o el infratratamiento de la depresión en los enfermos oncológicos está relacionada con una mayor discapacidad, una peor calidad de vida y una adherencia baja al tratamiento oncológico ${ }^{(17)}$. Los pacientes con cáncer avanzado tienen un riesgo aumentado de desarrollar síntomas depresivos. Para aquellos pacientes que desarrollen síntomas importantes de depresión están disponibles varias intervenciones eficaces. Si la depresión es muy severa o el paciente verbaliza ideación suicida debe ser remitido al psiquiatra para una evaluación más profunda, ya que la depresión es tratable incluso en el final de la vida ${ }^{(18)}$.

Casi todos los antidepresivos tienen la capacidad de mejorar al paciente. Pero para lograr esto, el paciente debe tomar la medicación y muchos de ellos no la toman si los efectos secundarios son mal tolerados. Estos autores proponen una estrategia en la farmacoterapia antidepresiva para intentar conseguir mayor éxito terapéutico:

- Primero debe elegirse según el perfil de efectos adversos, teniendo en cuenta los síntomas del paciente, de forma que se consiga el mayor beneficio posible. Por ejemplo la sedación o el estreñimiento que son efectos secundarios pueden ser útiles en un paciente con insomnio y diarrea.
- El segundo escalón es la evaluación del paciente cuando lleva un tiempo con la medicación. Si hay eficacia total y los efectos adversos no existen o son bien tolerados se mantiene el tratamiento.

- Si hay beneficio total pero no se toleran los efectos secundarios hay que tratar de aliviar éstos. Por ejemplo se intenta aliviar el estreñimiento con aporte de fibra.

- Si la mejoría es parcial y los efectos adversos son tolerables o no hay se mantendrá el tratamiento y se intentarán estrategias de potenciación. Por ejemplo añadiendo litio.

- Si la mejoría es parcial y los efectos adversos intolerables se cambiará de antidepresivo.

Recomendaciones prácticas para el uso de antidepresivos en el paciente oncológico(19)

Cuando la sintomatología depresiva está interfiriendo en el adecuado funcionamiento del paciente oncológico los antidepresivos son el tratamiento de primera línea, debiendo evitar el uso de benzodiacepinas como única estrategia farmacológica.

En este tipo de paciente el mayor riesgo de no adherencia terapéutica es en la $1^{\text {a }}$ semana de tratamiento, cuando los efectos adversos son superiores a la respuesta terapéutica. En este periodo es esencial dar adecuada información, aporte psicosocial y cuando sea necesario manejo farmacológico de estos efectos secundarios ${ }^{(20)}$.

El riesgo suicida existe en todos los pacientes con depresión severa. El riesgo está aumentado en aquellos con dolor crónico no controlado y depresión ${ }^{(21)}$.

\section{Elección del antidepresivo}

Esta debe basarse en la tolerancia y la dosis adecuada. En la elección de an- 
tidepresivo ha de considerarse: si ha habido respuesta previa a un antidepresivo, el conocimiento del medicamento por el médico, la preferencia del paciente y el coste.

La elección del antidepresivo en un paciente oncológico con depresión debe hacerse con cuidado debido a diversos factores. Uno de ellos son los efectos indeseables de los antidepresivos y otro la posible interacción de otros fármacos que toma el paciente con los antidepresivos ${ }^{(18)}$.

En la tabla 1 presentamos un resumen de los distintos antidepresivos y su indicación en pacientes con cáncer (Fuente: Modificada y adaptada de Grassi et al. ${ }^{(22)}$.

\section{Tabla 1. Resumen de los antidepresivos usados en pacientes con cáncer y sus características.}

\begin{tabular}{|c|c|c|c|c|}
\hline CLASE & ACCION & $\begin{array}{c}\text { EFECTOS } \\
\text { SECUNDARIOS } \\
\text { POSIBLES DESVENTAJAS } \\
\end{array}$ & $\begin{array}{c}\text { EFECTOS } \\
\text { SECUNDARIOS } \\
\text { POSIBLES VENTAJAS }\end{array}$ & $\begin{array}{l}\text { USO EN PACIENTES } \\
\text { CON CANCER }\end{array}$ \\
\hline \begin{tabular}{|c|} 
ANTIDEPRESIVOS \\
TRICÍCLICOS \\
Amitriptilina \\
Clorimipramina Imipramina.
\end{tabular} & \begin{tabular}{|c|} 
Inhibidor de \\
serotonina (5-HT) \\
y recaptación de \\
noradrenalina (NA) \\
Anti alfa 1 \\
Antihistamínico \\
Antimuscarínico
\end{tabular} & $\begin{array}{c}\text { Estreñimiento, boca } \\
\text { seca, retención urinaria, } \\
\text { alteración de la memoria. } \\
\text { Mareo, hipotensión } \\
\text { ortostática, somnolencia, } \\
\text { taquicardia refleja, } \\
\text { hipotensión }\end{array}$ & $\begin{array}{l}\text { Acción sobre el sueño } \\
\text { Acción sobre el dolor }\end{array}$ & $\begin{array}{l}\text { Generalmente } \\
\text { no es utilizado } \\
\text { por el riesgo de } \\
\text { efectos colaterales } \\
\text { antimuscarínicos. Si } \\
\text { es necesario el uso, } \\
\text { con precaución. }\end{array}$ \\
\hline $\begin{array}{l}\text { INHIBIDORES DE LA } \\
\text { RECAPTACIÓN DE } \\
\text { SEROTONINA (ISRS) } \\
\text { Fluoxetina Fluvoxamina } \\
\text { Escitalopram Citalopram } \\
\text { Sertralina Paroxetina } \\
\end{array}$ & $\begin{array}{l}\text { Inhibe la } \\
\text { recaptación de } \\
5-\mathrm{HT}\end{array}$ & $\begin{array}{c}\text { Disfunción sexual } \\
\text { (5-HT2A). Efectos } \\
\text { gastrointestinales } \\
\text { (nausea, vomito, diarrea) } \\
\text { (5HT3) }\end{array}$ & $\begin{array}{c}\text { Algunos más sedativos } \\
\text { que otros (ej.- } \\
\text { Citalopram) }\end{array}$ & $\begin{array}{l}\text { Uso regular con } \\
\text { la excepción de } \\
\text { Fluvoxamina (alta } \\
\text { interacción CYP). } \\
\text { Paroxetina interfiere } \\
\text { con tamoxifeno. } \\
\end{array}$ \\
\hline $\begin{array}{l}\text { INHIBIDORES DE LA } \\
\text { RECAPTACION DE } \\
\text { NORADRENALINA (IRN) } \\
\text { Reboxetina }\end{array}$ & \begin{tabular}{|c|} 
Inhibe la \\
recaptación de NA \\
Leve efecto \\
antimuscarínico \\
\end{tabular} & $\begin{array}{c}\text { Disminuye la tensión } \\
\text { arterial, mareo. } \\
\text { Posible sequedad de } \\
\text { boca, retención urinaria }\end{array}$ & $\begin{array}{l}\text { Mejora funciones } \\
\text { motoras y cognitivas }\end{array}$ & $\begin{array}{l}\text { No se usa de forma } \\
\text { rutinaria }\end{array}$ \\
\hline \begin{tabular}{|c|} 
INHIBIDORES DE \\
LA RECAPTACION \\
DE SEROTONINA Y \\
NORADRENALINA (IRSN) \\
Venlafaxina Desvenlafaxina \\
Duloxetina \\
\end{tabular} & $\begin{array}{l}\text { Inhibe la } \\
\text { recaptación de } \\
5-\mathrm{HT} \text { y la de NA }\end{array}$ & $\begin{array}{l}\text { Posible riesgo de } \\
\text { hipertensión }\end{array}$ & Acción sobre el dolor & $\begin{array}{c}\text { Más frecuentemente } \\
\text { usados }\end{array}$ \\
\hline $\begin{array}{c}\text { INHIBIDORES DE } \\
\text { LA RECAPTACIÓN } \\
\text { DE DOPAMINA Y } \\
\text { NOREPINEFRINA (IRDN) } \\
\text { Bupropion }\end{array}$ & $\begin{array}{c}\text { Inhibe la } \\
\text { recaptación de } \\
\text { dopamina (DA) } \\
\text { y NA }\end{array}$ & $\begin{array}{c}\text { Ansiedad } \\
\text { Activación psicomotora }\end{array}$ & $\begin{array}{c}\text { Aumenta la } \\
\text { concentración y la } \\
\text { atención } \\
\text { Disminuye la fatiga }\end{array}$ & $\begin{array}{c}\text { Algunos datos } \\
\text { en pacientes } \\
\text { con fatiga o en } \\
\text { fases avanzadas. } \\
\text { Control riesgo de } \\
\text { convulsiones } \\
\end{array}$ \\
\hline $\begin{array}{c}\text { AGENTE ESPECIFICO } \\
\text { SEROTONINÉRGICO Y } \\
\text { ADRENÉRGICO } \\
\text { Mirtazapina } \\
\end{array}$ & $\begin{array}{c}\text { Incrementa la } \\
\text { actividad de } 5-\mathrm{HT} \\
\text { y NA }\end{array}$ & Somnolencia & $\begin{array}{c}\text { Aumenta apetito y } \\
\text { ganancia ponderal. } \\
\text { Somnolencia (útil en } \\
\text { insomnio) }\end{array}$ & $\begin{array}{c}\text { Más frecuentemente } \\
\text { usado. Control } \\
\text { posible(rara) } \\
\text { neutropenia }\end{array}$ \\
\hline $\begin{array}{c}\text { INHIBIDOR DE LA } \\
\text { RECAPTACION/ } \\
\text { ANTAGONISTA 2 DE LA } \\
\text { SEROTONINA } \\
\text { Trazodona, Nefadozona }\end{array}$ & $\begin{array}{c}\text { Incrementa } \\
\text { actividad de 5-HT }\end{array}$ & & $\begin{array}{c}\text { Acción sobre el sueño } \\
\text { Efectos reportados en } \\
\text { dolor }\end{array}$ & $\begin{array}{c}\text { Usados en el } \\
\text { pasado. Nefadozona } \\
\text { puede causar } \\
\text { problemas hepáticos }\end{array}$ \\
\hline $\begin{array}{c}\text { PSICOESTIMULANTES } \\
\text { DAnfetamina Metilfenidato } \\
\text { Modafinilo }\end{array}$ & $\begin{array}{l}\text { Incrementa } \\
\text { actividad de DA }\end{array}$ & $\begin{array}{l}\text { Inquietud, agitación, } \\
\text { insomnio, pesadillas, } \\
\text { psicosis, anorexia, } \\
\text { arritmia, taquicardia, } \\
\text { hipertensión, tolerancia, } \\
\text { dependencia, } \\
\text { convulsiones } \\
\end{array}$ & $\begin{array}{l}\text { Efecto rápido } \\
\text { Acción sobre el dolor }\end{array}$ & $\begin{array}{c}\text { Usado } \\
\text { especialmente en } \\
\text { pacientes terminales }\end{array}$ \\
\hline
\end{tabular}


Interacciones farmacológicas

Las interacciones farmacológicas entre antidepresivos (AD) y antineoplásicos (AN) pueden comprometer la efectividad de los antineoplásicos e incrementar su toxicidad con las obvias implicaciones en el pronóstico. La información disponible sobre la metabolización de $\mathrm{AD}$ y $\mathrm{AN}$, con énfasis en el sistema del citocromo CYP 450, facilita la predicción en la interacción farmacológica, que podría ser evitada si esta fuera indeseable ${ }^{(23)}$. Las interacciones farmacocinéticas de los AD son poco probables con busulfan, clorambucil, melfalán, tioguanina, cisplatino, carboplatino, oxaliplatino, mercaptopurina, 5-fluorouracilo, estramustina, daunorubicin, temozolomida, mecloretamina, doxorubicina, epirubicina, vorinostat.

La mayoría de los antineoplásicos sujetos a metabolización por el citocromo CYP 450 3A4 deberían ser utilizados con precaución en el uso concomitante con inhibidores de esta isoenzima como son la Fluoxetina, Sertralina y Paroxetina. Fluvoxamina, Escitalopram, Citalopram, Venlafaxina, Mirtazapina son más seguros en estos pacientes ya que el potencial inhibidor del CYP 450 es mínimo(23).

Hay que extremar la precaución en el uso simultáneo de antidepresivos de acción dual (en particular Venlafaxina) y Tramadol por la posibilidad de producir un síndrome serotoninérgico ${ }^{(24)}$.

La elevada complejidad que presentan los pacientes oncológicos en su tratamiento, por un lado el que implica a su trastorno de base unido al tratamiento de los efectos colaterales (efectos secundarios de la quimioterapia y/o radioterapia) y no por ello menos importante, genera en los pacientes dificultades para comprender y aceptar una situación que amenaza su supervivencia. En la actualidad, se están realizando múltiples investigaciones para tratar de mejorar la calidad de vida de di- chos pacientes. Por ello, el interés de este estudio es analizar y comparar los resultados de las publicaciones realizadas en los últimos 15 años sobre la eficacia de diferentes fármacos antidepresivos en pacientes con comorbilidad de depresión y cáncer.

\section{METODOLOGÍA}

Se realizó una búsqueda bibliográfica en la base de datos PubMed mediante los términos: <cancer AND depression treatment AND antidepressant>. De los 795 artículos encontrados inicialmente se excluyeron aquellos artículos publicados en lenguas distintas al inglés y castellano.

El período analizado comprende desde el enero de 1998 hasta enero de 2016. Los criterios de inclusión son: estudios realizados en humanos, estudios tipo ensayo clínico y que aparecieran a texto completo. De un total de 99 artículos restantes, se fueron seleccionando aquellos que estudiaban la efectividad de diferentes terapias farmacológicas usadas en pacientes con cáncer y depresión (se excluyeron las terapias no farmacológicas) y aquellas que no analizaban población oncológica con síntomas depresivos.

\section{RESULTADOS}

Analizamos los resultados encontrados a lo largo de 36 artículos que analizan los resultados de una intervención farmacológica mediante diseños de ensayo clínico (uso de un antidepresivo respecto a un placebo o bien la comparación de un antidepresivo respecto a otro) en población oncológica con diferentes grados de sintomatología depresiva (ver la tabla 2). En 23 de ellos la muestra fue aleatorizada para permitir la reducción de los sesgos de selección. En 24 de los estudios la muestra está compuesta exclusivamente por mujeres, sin embargo el resto de 


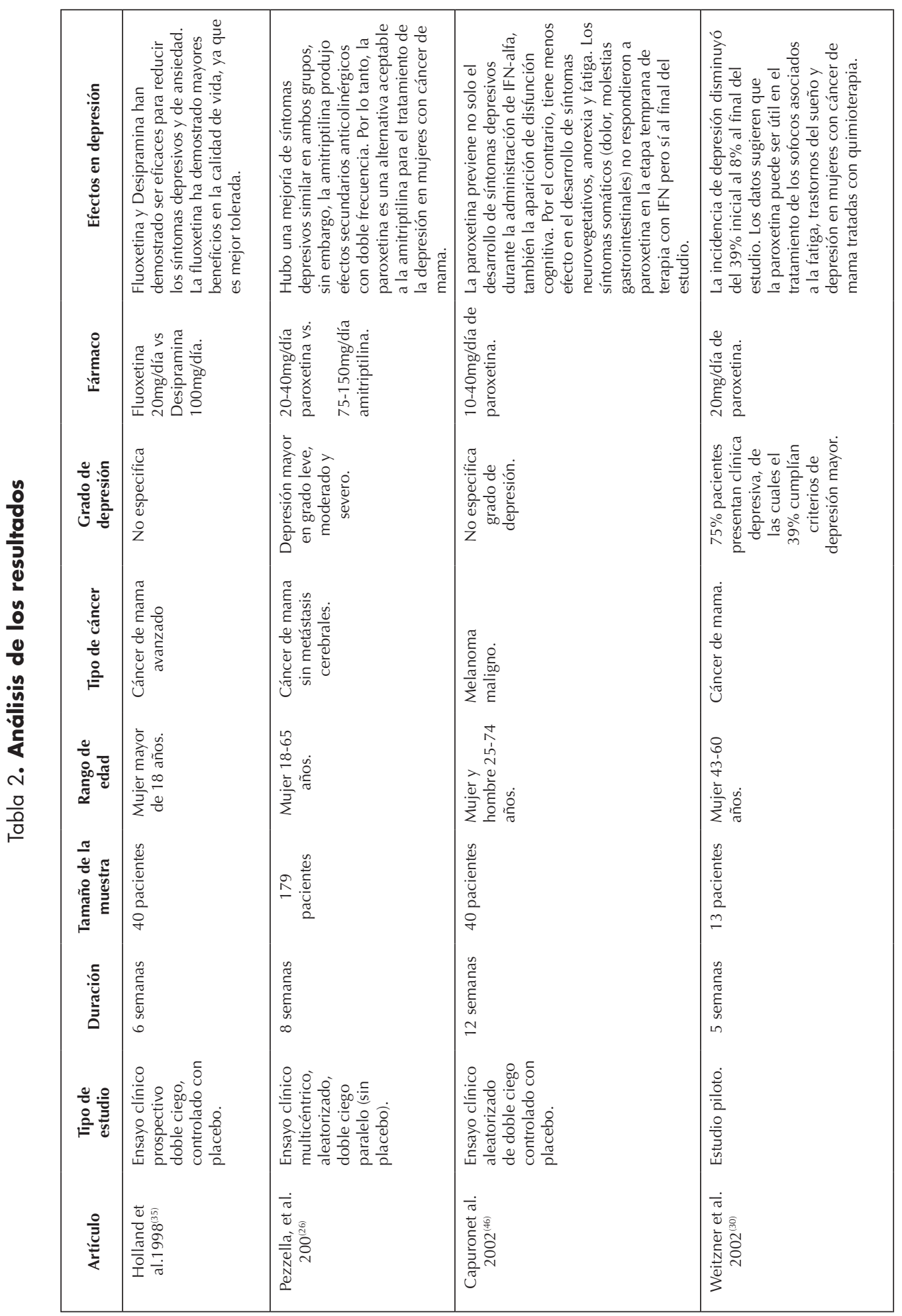




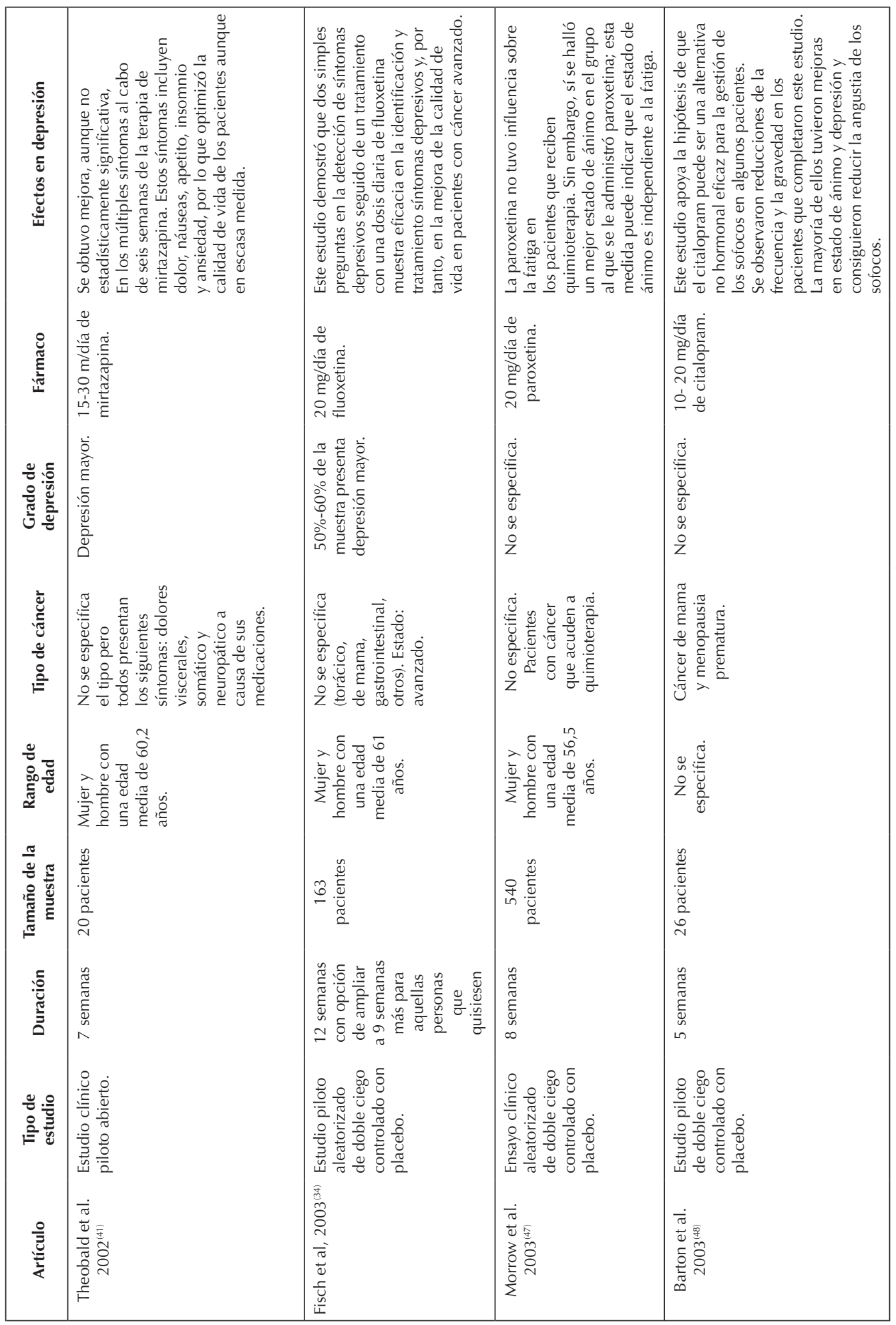




\begin{tabular}{|c|c|c|c|c|c|c|}
\hline 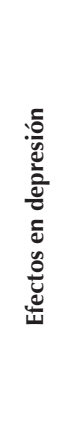 & 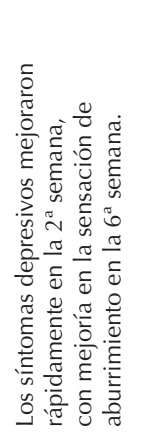 & 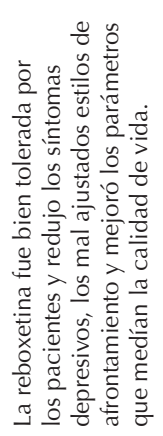 & 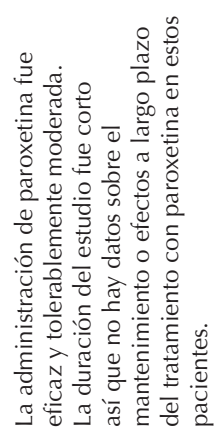 & 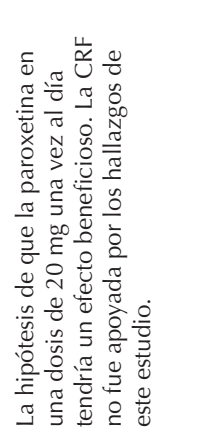 & 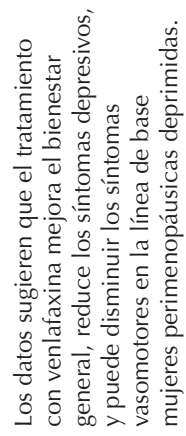 & 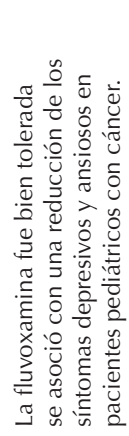 \\
\hline 龸 & 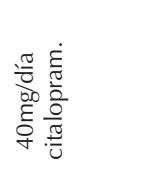 & 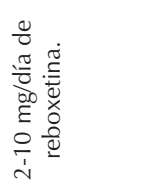 & 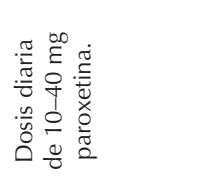 & 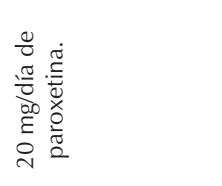 & 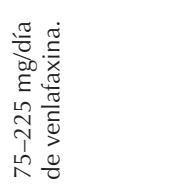 & 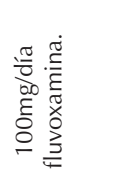 \\
\hline 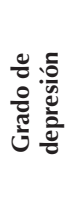 & 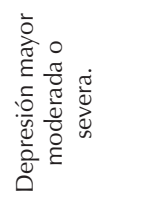 & 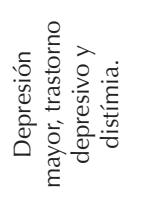 & 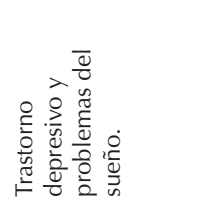 & 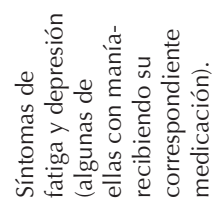 & 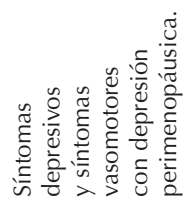 & 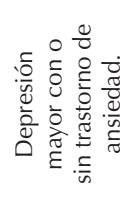 \\
\hline 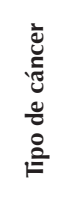 & 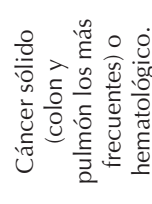 & 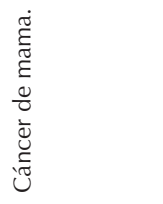 & 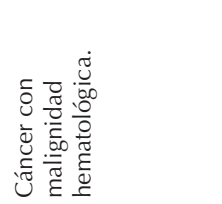 & 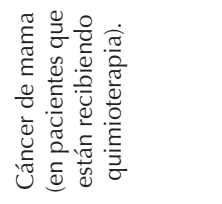 & 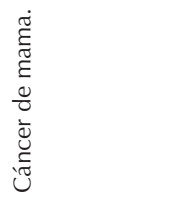 & 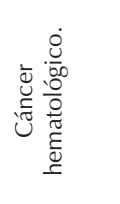 \\
\hline 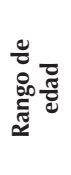 & 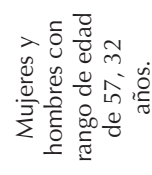 & 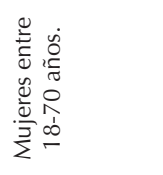 & 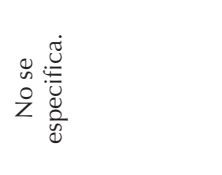 & 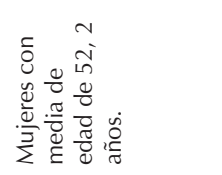 & 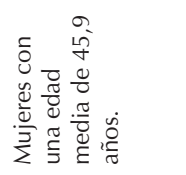 & 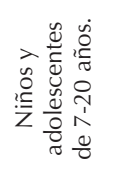 \\
\hline 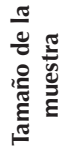 & 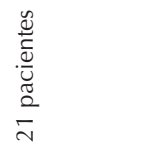 & 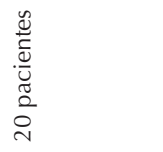 & 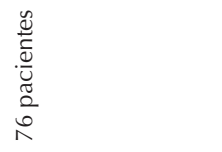 & 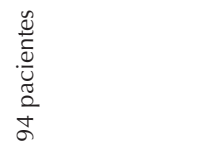 & 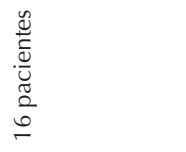 & 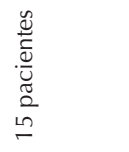 \\
\hline 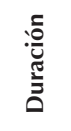 & 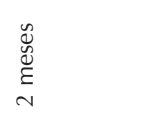 & 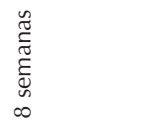 & 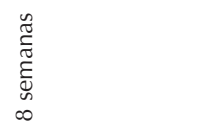 & 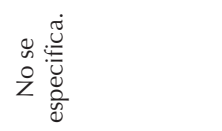 & 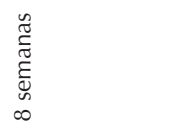 & 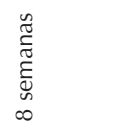 \\
\hline 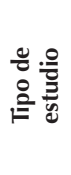 & 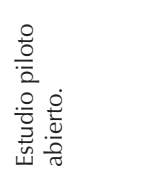 & 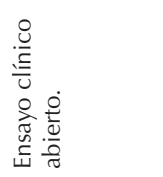 & 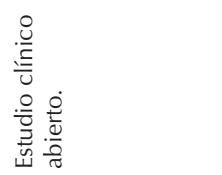 & 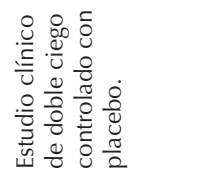 & 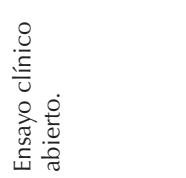 & 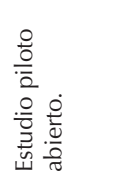 \\
\hline 畩 & 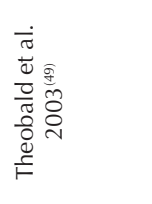 & 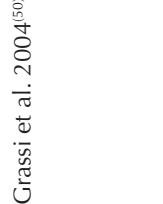 & 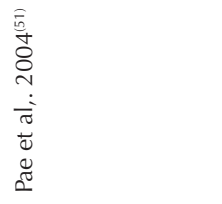 & 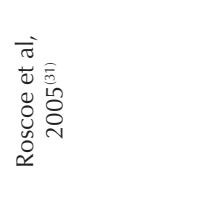 & 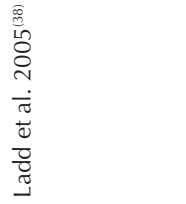 & 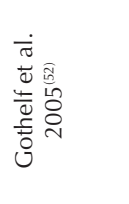 \\
\hline
\end{tabular}




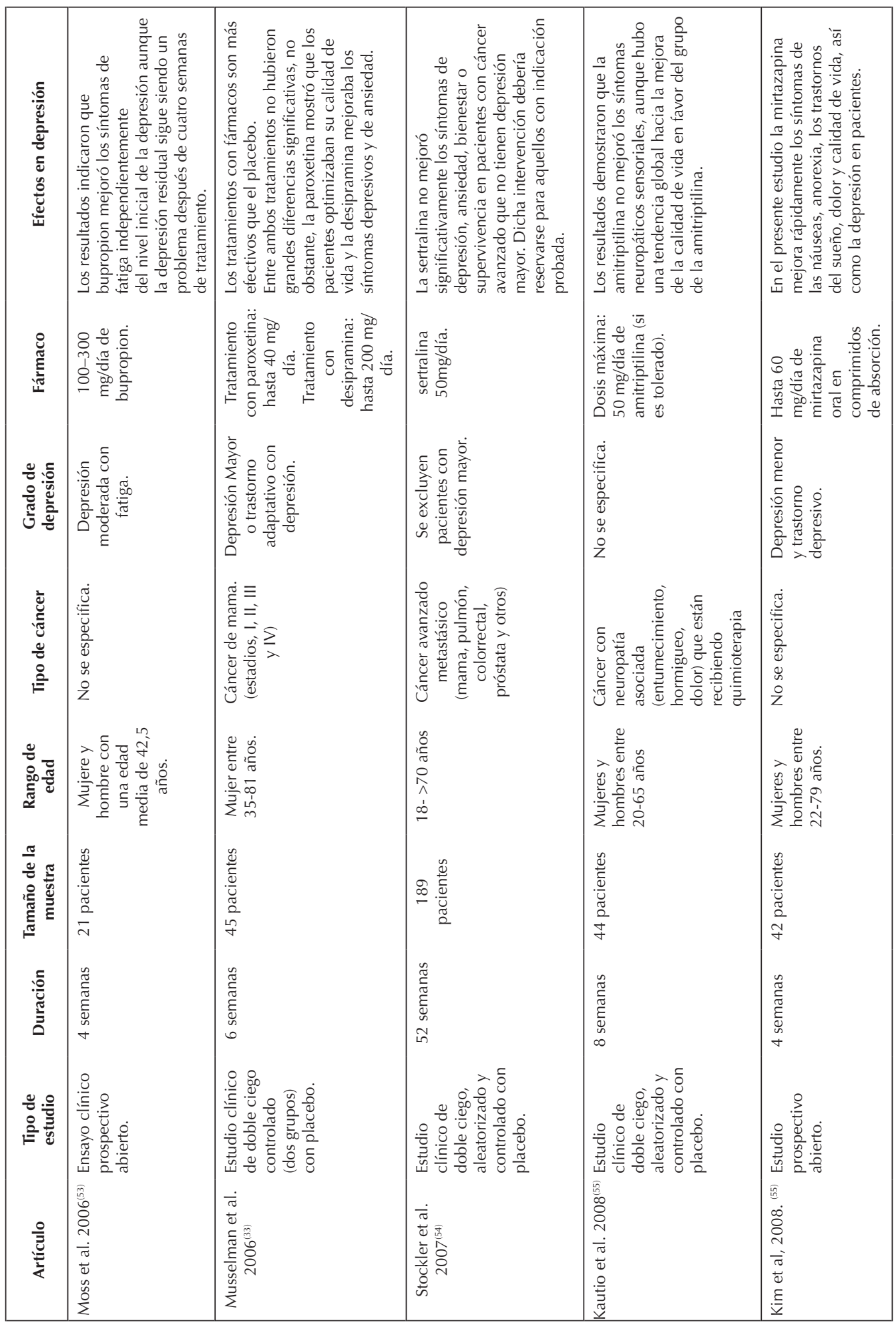




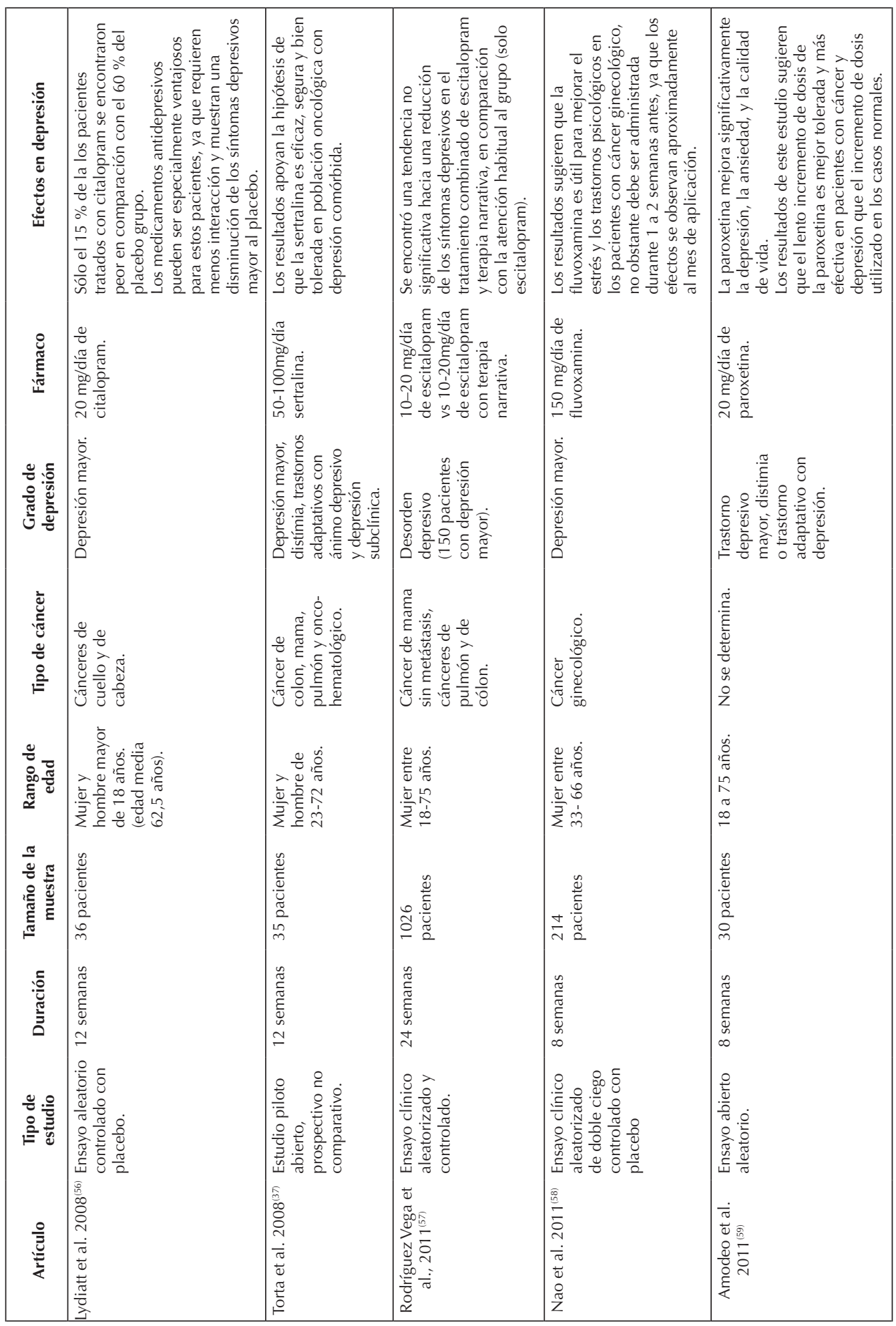




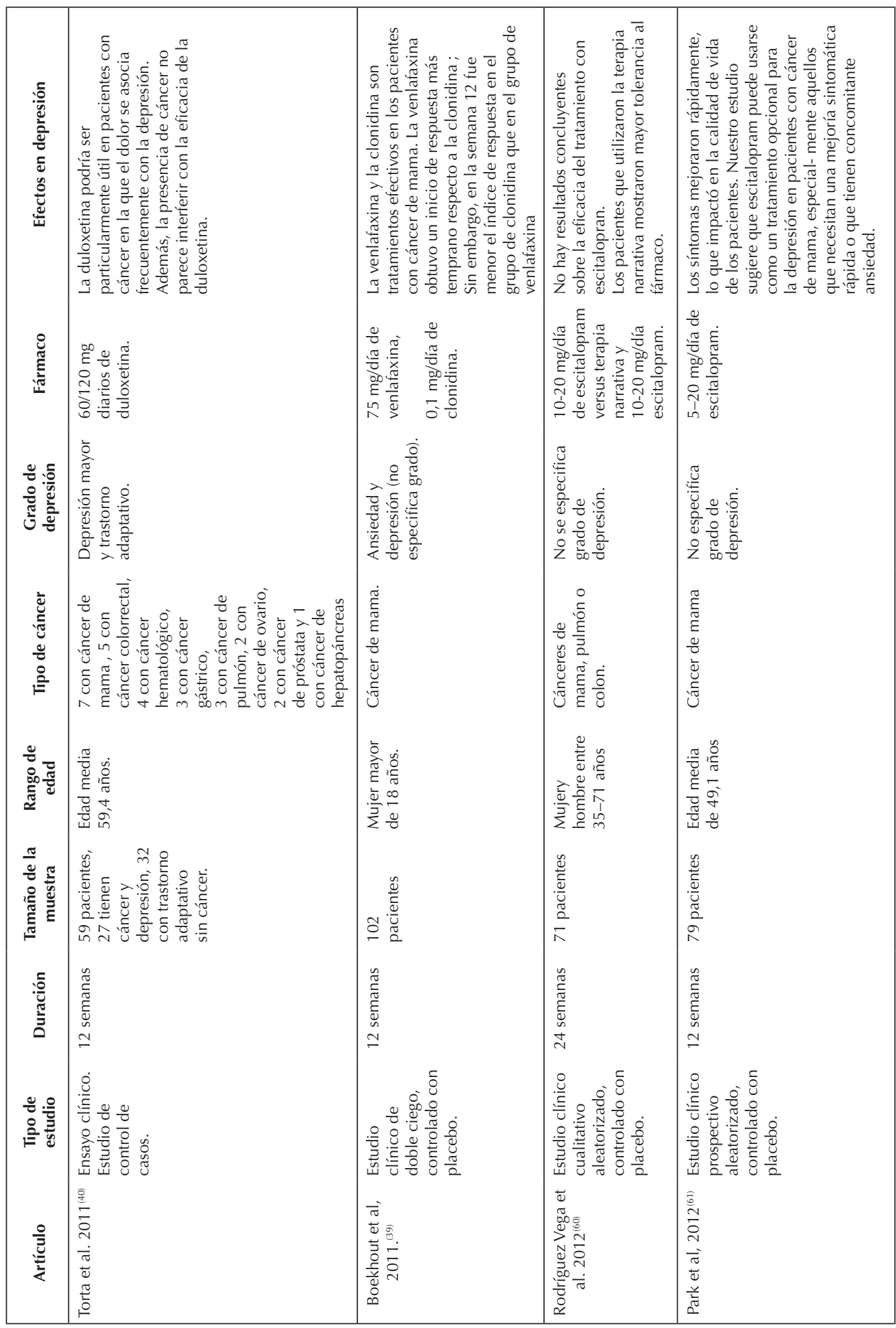




\begin{tabular}{|c|c|c|c|c|}
\hline 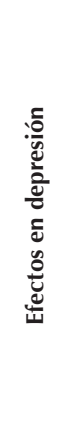 & 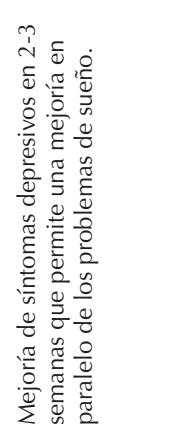 & 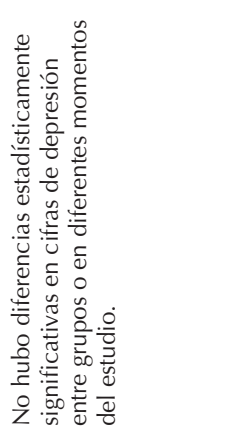 & 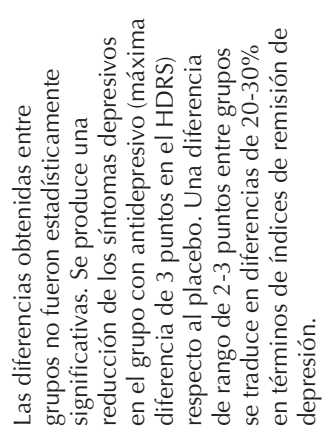 & 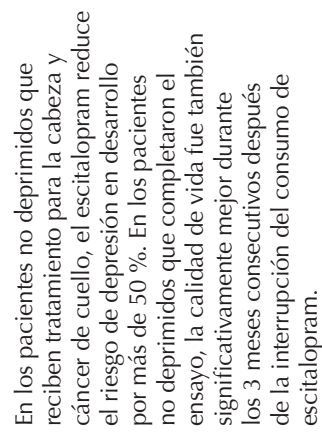 \\
\hline 龸 & 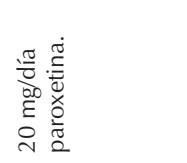 & 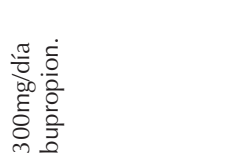 & 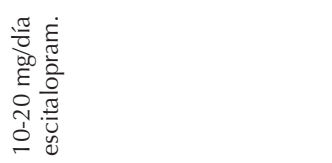 & 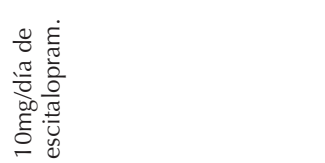 \\
\hline 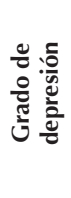 & 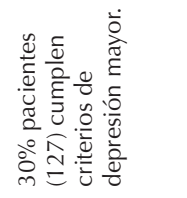 & 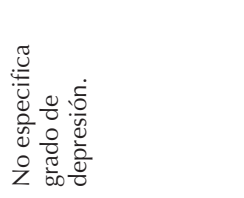 & 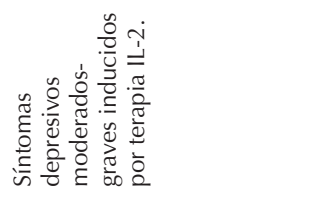 & 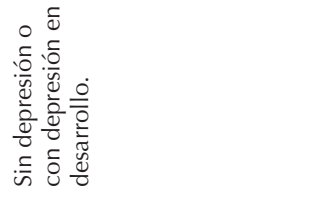 \\
\hline 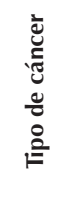 & 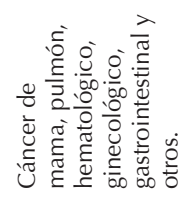 & 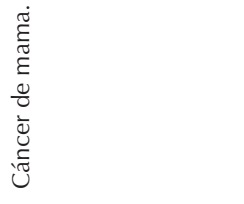 & 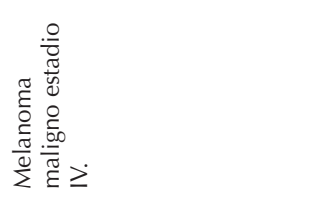 & 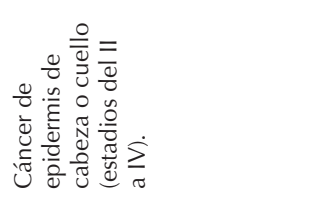 \\
\hline 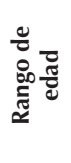 & 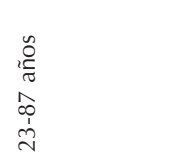 & 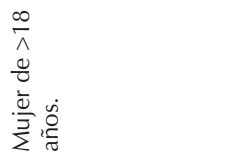 & $\begin{array}{l}\dot{0} \\
\dot{0} \\
\text { c } \\
\infty \\
0 \\
0 \\
\dot{N}\end{array}$ & 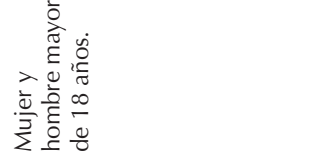 \\
\hline 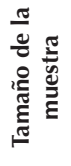 & 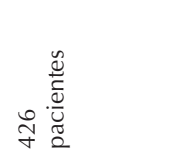 & 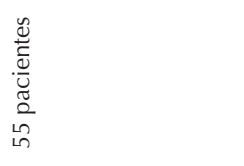 & 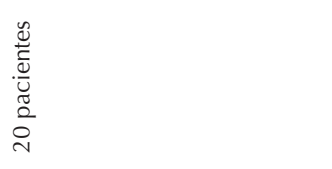 & 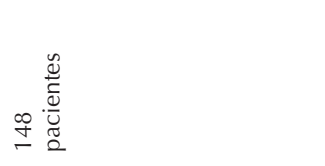 \\
\hline 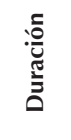 & 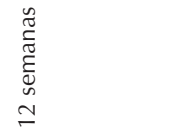 & 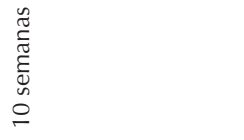 & 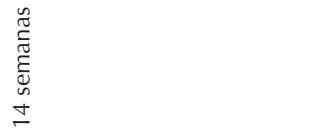 & 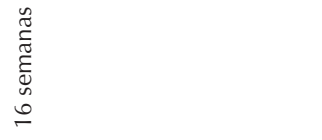 \\
\hline 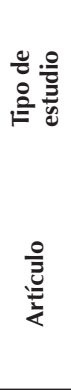 & 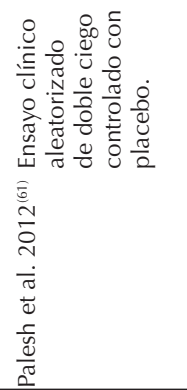 & 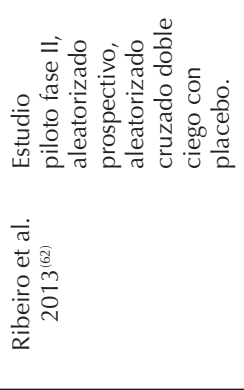 & 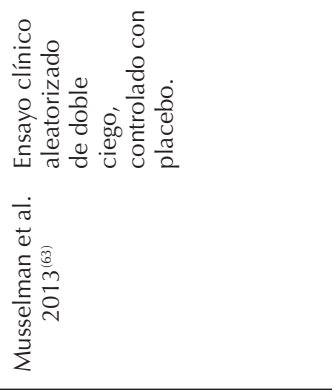 & 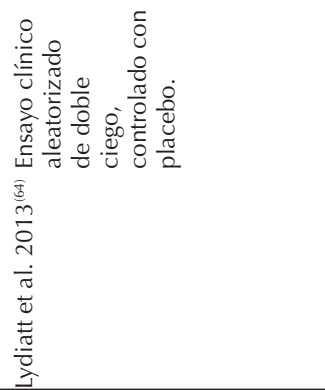 \\
\hline
\end{tabular}




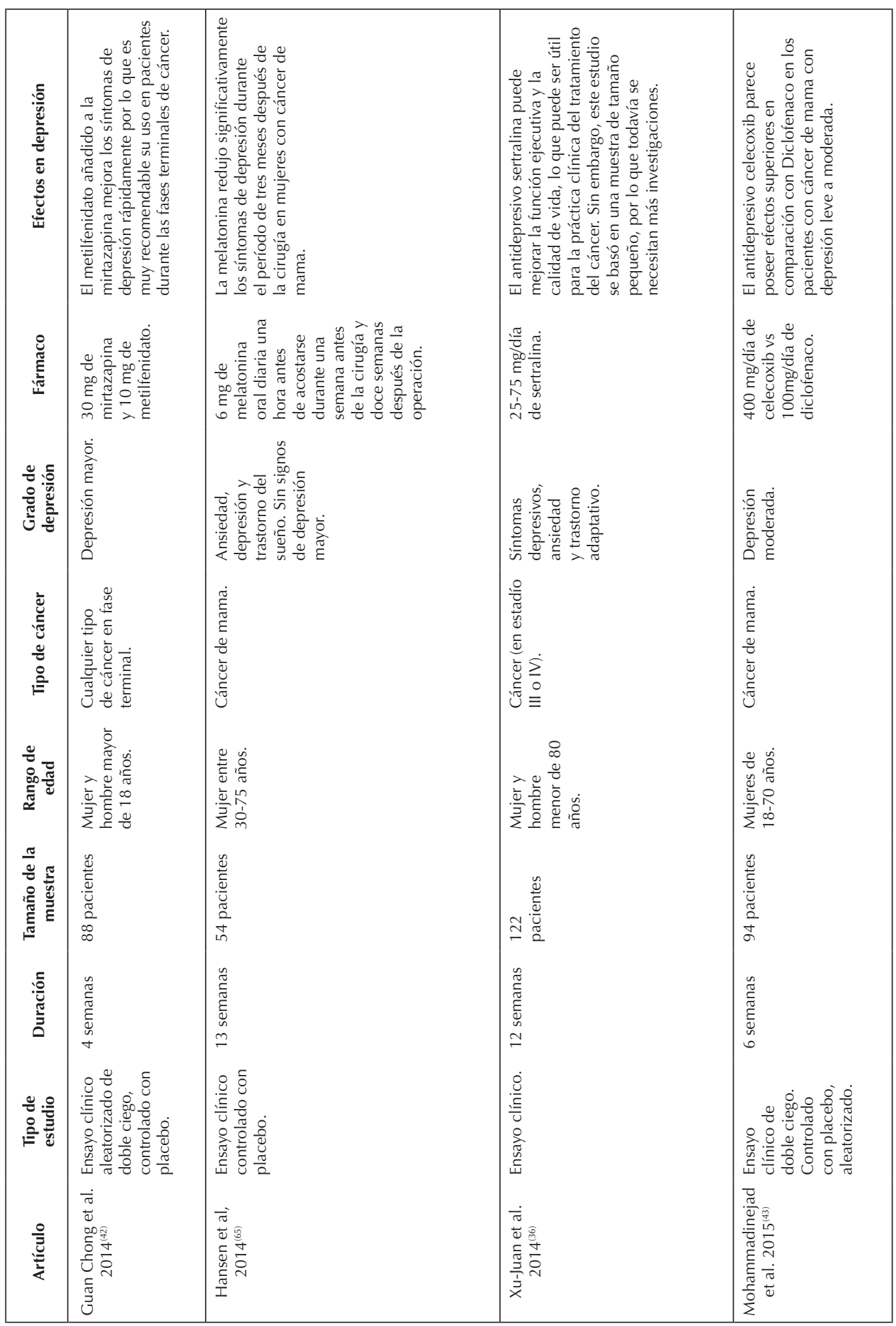


estudios es más heterogéneo en cuanto al sexo de la muestra pero con mayor similitud en cuanto al diagnóstico de cáncer y grados de sintomatología depresiva. La edad de los pacientes estudiados está comprendida entre los 18 y 80 años, salvo en uno de los estudios en que se estudia población infantil y adolescente con edades comprendidas entre los 7-20 años. Los antidepresivos utilizados fueron ISRS en su mayoría (casi en el 50\% de los artículos). 9 estudios analizaron el efecto de la paroxetina a dosis de entre 20-40mg/día, obteniéndose resultados beneficiosos en la depresión sobre todo por su rapidez de acción. Tres estudios analizaron la eficacia de citalopram a dosis de 10-40mg/día, dos analizaron la fluoxetina a dosis de 20mg/ día, otros dos escitalopram (en este caso uno de los estudios encontró datos contradictorios frente a placebo), otro sertralina a dosis de 50-75mg/día recomendable, siempre que sea a esta dosis o mayor $y$ fluvoxamina también útil tanto en adultos como en población pediátrica. A grandes rasgos y como analizaremos en detalle gracias a la tabla de resultados, son muy bien tolerados y tienen efectividad demostrada para el tratamiento de síntomas depresivos en población oncológica.

Tres de los estudios analizan fármacos de acción dual, encontrándose beneficioso por su efecto de respuesta temprano y perfil beneficioso al actuar en síntomas vasomotores que acompañan con frecuencia a los síntomas depresivos (mayoritariamente en población femenina).

La mirtazapina es analizada en 3 artículos, siendo una opción muy interesante por su efectividad en síntomas derivados del cáncer como son el dolor, las náuseas, la falta de apetito y el insomnio entre otros.

El bupropion es un antidepresivo que se ha visto que es útil para el tratamiento de la fatiga en mujeres con depresión (aunque existe el riesgo de no mejorar sintomatología depresiva residual).
La amitriptilina, fármaco usado con más frecuencia en el pasado dada su mala tolerancia por los elevados efectos secundarios también ha sido estudiado en por Kautio et al. ${ }^{(25)}$ y Pezzella et al. ${ }^{(26)}$ encontrándolo beneficioso si utilizamos su perfil anticolinérgico para mejorar síntomas como habíamos mencionado que ocurría con la mirtazapina. Por último, dos estudios analizan la eficacia de dos fármacos no antidepresivos que pueden ser útiles en la depresión (melatonina a dosis de 6mg/ día y Celecoxib en depresiones moderadas en mujeres con cáncer de mama). Los resultados de cada estudio se exponen en la tabla 2.

\section{DISCUSIÓN}

La depresión es un problema importante en los pacientes oncológicos, con una elevada prevalencia(27). El ánimo depresivo interfiere en la calidad de vida y en la adherencia al tratamiento oncológico, afectando al paciente globalmente (tanto a nivel físico, como psíquico y social ${ }^{(28)}$. No reconocer o no tratar la depresión aumenta no solo la morbilidad sino también la mortalidad del cáncer ${ }^{(27)}$.

Es fundamental el diagnóstico y tratamiento de la depresión en los pacientes oncológicos, ya que puede influir negativamente en aspectos como la duración del ingreso hospitalario, el autocuidado, el cumplimiento terapéutico, la calidad de vida y la supervivencia ${ }^{(29)}$. Por estos motivos, analizamos los resultados de varios estudios sobre la eficacia de antidepresivos de diferentes perfiles en muestras de pacientes oncológicos que padecen depresión en mayor o menor grado.

En el actual estudio se han analizado un total de 36 publicaciones que investigan la tolerabilidad y eficacia de los fármacos más utilizados en el tratamiento de la depresión en cualquiera de sus grados (grave, moderado y leve) en pacien- 
tes adultos oncológicos. Solo uno de los ensayos analiza los efectos causados en pacientes pediátricos.

Es importante destacar que hay una mayor proporción de artículos que analizan la eficacia de fármacos inhibidores de la recaptación de serotonina (ISRS). Según las guías de tratamiento de la depresión, es recomendable iniciar un fármaco de este perfil por su perfil de tolerabilidad y eficacia.

La paroxetina es el ISRS más analizado, siendo estudiada en nueve de los 35 artículos. De todos ellos, podemos destacar que en dosis diarias de 20-40 mg en pacientes con cáncer y depresión mayor se producen mejoría de síntomas, al igual que en pacientes que manifiestan problemas de sueño.

En uno de ellos ${ }^{(30)}$ se vio que el consumo del fármaco (a dosis de $20 \mathrm{mg} /$ día) durante la aplicación de quimioterapia aumentaba el estado de ánimo pero no influía en la fatiga. Además, este es apoyado por otro estudio ${ }^{(31)}$ en el que se encuentra una mejora de la depresión en aquellas pacientes con cáncer de mama, además de ser útil para la prevención de los sofocos asociados a la fatiga.

También destacar que en pacientes con cáncer unido a depresión mayor, distimia o trastorno adaptativo se producen mejoras en los síntomas depresivos, la ansiedad y los problemas de sueño en 2-3 semanas ${ }^{(32)}$.

Ahora bien, si observamos el trabajo de Musselaman et al. ${ }^{(33)}$, en el que se prueba la eficacia de la paroxetina (dosis máxima de $40 \mathrm{mg} /$ día) vs desipramina (dosis máxima de 200 mg/día) en pacientes con cáncer y depresión mayor o trastorno adaptativo con depresión concluimos que no existe una gran diferencia entre ambas. No obstante, la paroxetina mejora la calidad de vida mientras que la desipramina mejora los síntomas de ansiedad.

Por otra parte, en otro estudio Fisch ${ }^{(34)}$ se muestra que la fluoxetina a dosis de 20 $\mathrm{mg}$ /día mejora la calidad de vida de los pacientes con cáncer avanzado. Holland et al. ${ }^{(35)}$ encontraron beneficios en el uso de fluoxetina frente al uso de desipramina dado su mejor perfil de tolerancia siendo ambos antidepresivos útiles para reducir los síntomas ansiosos y depresivos.

Respecto al citalopram (uso de dosis de 10-40 mg/día) abordado en tres de los artículos revisados supone una alternativa no hormonal eficaz para la gestión de los sofocos y la disminución de la depresión y la angustia en pacientes con cáncer de mama y con menopausia. El citalopram produce mejoras en la segunda semana de tratamiento en pacientes con cáncer solido o hematológico y depresión mayor y moderada.

Sin embargo, con el escitalopram encontramos datos contradictorios. En un estudio $^{(36)}$ no se encontraron diferencias entre el fármaco y su placebo al evaluar su eficacia en el ánimo y en otro ${ }^{(37)}$ se encontró que mejoraba los síntomas depresivos rápidamente.

La sertralina en dosis de $50-75 \mathrm{mg} /$ día mejoraba la función ejecutiva en personas con cáncer en estadios III y IV, Ios síntomas depresivos, ansiedad y trastorno adaptativo. Sin embargo, en dosis menores y en pacientes con depresión menor y cáncer avanzado no reduce significativamente la depresión, la ansiedad o el bienestar general.

Por último, la fluvoxamina se ha encontrado que mejora el nivel de estrés y el trastorno psicológico en pacientes adultos oncológicos después de 6-8 semanas de tratamiento y en pacientes pediátricos con cáncer hematológico es un fármaco bien tolerado.

Analizando los resultados encontrados respecto a los fármacos de perfil dual (aquellos que actúan en serotonina y noradrenalina), vemos que la venlafaxina tiene un efecto de respuesta temprano, mejora el bienestar general y disminuye los síntomas vasomotores en mujeres peri- 
menopáusicas deprimidas ${ }^{(38)}$. No obstante, la clonidina aunque es más tardía en reaccionar da mayor respuesta que la anterior en ansiedad y depresión a partir de la $12^{\mathrm{a}}$ semana $^{(39)}$.

Por su parte, La duloxetina en pacientes con oncológicos que presentan dolor y depresión mayor es muy útil, específicamente en dosis de $60-120 \mathrm{mg} / \mathrm{día}^{(40)}$. Estos resultados nos permiten concluir respecto a los antidepresivos duales que son eficaces en depresión, por su rapidez de acción y por su utilidad en síntomas colaterales secundarios a ciertos cánceres.

El bupropion es un antidepresivo que actúa en noradrenalina y dopamina. Hemos encontrado un estudio que encuentra mejoría de los niveles de fatiga en pacientes con cáncer de mama y depresión pero después de 4 semanas de estudios aún quedan muestras de depresión residual.

La mirtazapina, sin embargo, es otro fármaco bastante estudiado en proporción al resto de antidepresivos (de hecho es analizada en 3 de los ensayos clínicos). Se ha encontrado que en pacientes con diagnóstico de depresión mayor mejora los síntomas de dolor, náuseas, apetito, insomnio y ansiedad, por lo que aumenta la calidad de vida en 6 semanas $^{(41)}$. Además, si se le añade al tratamiento metilfenidato ${ }^{(42)}$ aumenta la rapidez en la mejoría en los pacientes, por lo que es muy útil en aqueIlos que se encuentran en la fase terminal de sus cánceres.

La amitriptilina y la desipramina son fármacos de la familia de los antidepresivos tricíclicos, que como es sabido actúan en los receptores de serotonina, noradrenalina y dopamina (lo que le confiere una elevada potencia antidepresiva). Además, es capaz de actuar en otros receptores, lo que incrementa los efectos secundarios y disminuye en gran medida su tolerabilidad. Por ello, no es un perfil de antidepresivo utilizado de primera línea de tratamiento. Atendiendo a los estudios de Kautio et al. ${ }^{(25)}$ y Pezzella et al. ${ }^{(26)}$ y Holland et al. ${ }^{(35)}$ encontraron que proporciona mejoría en la calidad de vida en los pacientes, aunque contiene demasiados efectos secundarios anticolinérgicos y tampoco mejora los síntomas neuropáticos sensoriales (entumecimiento, hormigueo y dolor).

Por último, destacar que se ha realizado alguna publicación con otros fármacos que no son estrictamente antidepresivos pero que pueden ayudar a mejorar síntomas que acompañan a la depresión ${ }^{(43)}$. En uno de los artículos analizados en el presente trabajo se ha encontrado que la melatonina (dosis de $6 \mathrm{mg}$ /día durante tres meses) reduce los síntomas de depresión mayor en las pacientes con cáncer de mama ${ }^{(44)}$. Por otro lado, el celecoxib parece ser más eficaz que el Diclofenaco en pacientes con depresiones moderadas y cáncer de mama de acuerdo con otro de los estudios realizados $^{(43)}$.

La principal limitación encontrada está directamente relacionada con los fármacos utilizados como objeto de análisis a lo largo de los estudios. Encontramos mayor número de publicaciones de fármacos que actúan en receptores serotoninérgicos, en probable relación con su mayor uso y tolerabilidad. Sin embargo, otros fármacos han ido dejándose de utilizar progresivamente. Este dato es interesante, ya que, aunque no encontremos estudios que desaconsejen su uso, la menor utilización de estos esté probablemente relacionada con peor tolerancia y eficacia. Respecto a las muestras analizadas, otra limitación es la elevada proporción de artículos que analizan como población diana a las mujeres diagnosticadas de cáncer de mama (no siendo el cáncer más prevalente). Sería interesante poder ampliar dichos estudios a otros perfiles de pacientes diferentes al ya expuesto.

Son necesarios más estudios para evaluar la eficacia de las intervenciones antidepresivas, identificar estrategias que me- 
joren las tasas de detección y que evalúen la eficacia y tolerabilidad de nuevos tratamientos antidepresivos y el uso de estrategias combinadas ${ }^{(45)}$.

Dicho esto, es recomendable que la prescripción del antidepresivo sea realizada por un psiquiatra teniendo en cuenta las experiencias farmacológicas previas, la situación clínica del paciente, sus comorbilidades y las potenciales interacciones farmacológicas.

Futuros estudios deberían cubrir la falta de investigación existente que compare la efectividad de los diferentes fármacos en situaciones clínicas diferentes. Son necesarios más estudios controlados sobre el uso específico de antidepresivos en los pacientes oncológicos.

\section{REFERENCIAS BIBLIOGRÁFICAS}

1. Ritterband LM, Spielberg CD. Depression in a cancer population. J Clin Psychol Med Setings 2001;8:85-93. Doi: 10.1023/A:1009551809695

2. Akechi T, Nakano T, Akizuki N, Okamura M, Okamura M, Sakuma K, et al. Somatic symptoms for diagnosing major depression in cancer patients. Psychosomatics 2003; 44:244-8. Doi: 10.1176/appi. psy.44.3.244

3. Coyne JC, Thompson R, Klinkman MS, Nease Jr DE. Emotional disorders in primary care. J Cons Clin Psychol 2002;70:798809. Doi: 10.1037/0022-006X.70.3.798

4. Park EM, Raddin RS, Nelson KM, Hamer RM, Mayer DK, Bernard SA et al. Conducting an antidepressant clinical trial in oncology: challenges and strategies to address them. Gen Hosp Psychiatry 2014;36:474-6. Doi: 10.1016/j.genhosppsych.2014.05.007

5. Massie MJ. Prevalence of depression in patients with cancer. JNCl Monogr 2004;32:57-71.

6. Trask, P.C. Assessment of depression in cancer patient. Journal of the National Can- cer Institute Monographs.2004;32:80-92. Doi:10.1093/jncimonographs/lgh

7. Pousa V, Miguelez A, Hernandez M, Gonzalez MA. Depresión y cáncer: una revisión orientada a la práctica clínica. Rev Colomb Cancerol 2015;19: 166-72. Doi:10.1016/jrccan.2015.04.005

8. Gil Moncayo FL, Costa Requena G, Pérez FJ, Salamero M, Sánchez N, Sirgo, A. Adaptación psicológica y prevalencia de trastornos mentales en pacientes con cáncer. Med Clín (Barc) 2008; 130:90-2. Doi: $10.1157 / 13115354$

9. Weinbeger MI, Roth AJ, Nelson CJ. Untangling the complexities of depression diagnosis in older cancer patients. Oncologist 2009;14:60-6. Doi: 10.1634/theoncologist.2008-0147

10. Pelletier G. Verhoef MJ, Khatrei N, Hagan N. Quality of life in brain tumor patients: the relatives contributions of depression, fatigue, emotional distress and existential issues. J Neuroncol 2002;57:41-9

11. Hernandez M, Cruzado JA, Prado C, Hernandez C, Gonzalez MA et al. Salud mental y malestar emocional en pacientes con cancer. Psiconcología 2012;9:23357. Doi: 10.5209/rev_PSIC.2013.v9.n23.40895

12. Roca BM, Arroyo MB. Depresión y enfermedad médica. En Roca BM, Arroyo MB, editores. Trastornos depresivos en patologías médicas. Barcelona: Masson S.A., 1996

13. Palmer SC, Coyne JC. Screeening for depression in medical care: pitfalls, alternatives, and revised priorities. J Psychosom Res 2003;54:279-87. Doi:10.1016/S00223999(02)00640-2

14. Voderamier A, Linden W, Siu C. Screening for emotional distress in cancer patients: a sistematic review of assessment instrument. J Natl Cancer Inst 2009;101:146488. Doi: 10.1093/jnci/djp336.

15. Valencia SC. Depresión en personas diagnosticadas con cáncer. Diversitas: perspectivas en Psicología. 2006; 2:241-57 
16. Hart SL, Hoyt MA, Diefenbach M, Anderson DR, Kilbourn KM, Craft LL, et al. Meta-analysis of efficacy of interventions for elevated depressive symptoms in adults diagnosed with cancer. J Natl Cancer Inst 2012;104:990-10. Doi: 10.1093/jnci/ djs256

17. Caruso R, Grassi, Nanni MG, Riba M. Psychopharmacology in Psycho-Oncology. Curr Psychiatry Rep 2013;5:393. Doi: 10.1007/s11920-013-0393-0.

18. Winell J, Roth AJ. Depression in cancer patients. Oncology 2004;18:1554-60.

19. Torta RGV, Leraci, V. Pharmacological management of depression in patients with cancer: practical considerations. Drugs 2013,73:1131-45. Doi: 10.1007/s40265013-0090-7.

20. Aikens JE, Nease DE Jr, Nau DP, Klinkman MS, Schwenk TL Adherence to maintenance phase antidepressant medication as a function of patient beliefs about medication. Ann Fam Med 2005,3:23-30. Doi:10.1370/afm.238

21. Kanzler KE, Bryan CJ, McGeary DD, Morrow CE. Suicidal ideation and perceived burdensomeoneness in patients with cronic pain. Pain Pract 2012;12:602-9. Doi: 10.1111/j.1533-2500.2012.00542.x

22. Grassi L, Nanni MG, Uchitomi Y, Riba M. Pharmacotherapy of depression in people with cancer. En Kissane DW, Maj M, Sartorius N, editors. Depression and Cancer. Chichester: Wiley 2011.p.151-76.

23. Miguel C, Alburquerque E. Drug interaction in Psycho-Oncology: Antidepressants and antineoplastics. Pharmacology 2011, 88:333-9. Doi: 10.1159/000334738

24. Scalabrino A, Airoldi M, Stanizzo MR, Torta $R$, Transmethylants in oncology: antidepressant effectiveness. Ann Oncol Suppl 2008;19:17.

25. Kautio A-L, Haanpää M, Saarto T, Kalso E. Amitriptyline in the treatment of chemotherapy-induced neuropathic symptoms. J Pain Symptom Manage 2008;35:31-9. Doi. 10.1016/j.jpainsymman.2007.02.043
26. Pezzella G, Moslinger-Gehmayr R, Contu A. Treatment of depression in patients with breast cancer: A comparison between paroxetine and amitriptyline. Breast Cancer Res Treat 2001:70:1-10.

27. Onitilo AA, Nietert PJ, Egede LE. Effect of depression on all cause mortality in adults with cancer and differential Effects by cancer site. Gen Hosp Psychiatry 2006;28:396-402. Doi:10.1016/j.genhosppsych.2006.05.006

28. Fernández Corcuera, P, Dolz, M, Rojo, JE. Uso de psicofármacos en el enfermo oncológico. En: Gil F, editor. Manual de Psico-Oncología. Madrid: Nova Sidonia 2000, p. 75-91.

29. Mc Daniels JS, Nemeroff, CB. Depression in the cancer patient. Diagnostic, biological, and treatment aspects. En: Chapman CR, Foley KM, editors. Current and Emerging Issues in Cancer Pain. Nueva York: Raven Press 1993.p.1-19.

30. Weitzner MA, Moncello J, Jacobsen PB., Minton SA. Pilot trial of paroxetine for the treatment of hot flashes and associated symptoms in women with breast cancer. J Pain Symptom Manage 2002;23: 337-45. Doi:10.1016/S0885-3924(02)00379-2

31. Roscoe JA, Morrow GR, Hickok JT, Mustian KM, Griggs JJ, Matteson SE, et al. Effect of paroxetine hydrochloride (Paxil) on fatigue and depression in breast cancer patients receiving chemotherapy. Breast Cancer Res Treat 2005;89: 243-9. Doi: 10.1007/s10549-004-2175-1

32. Palesh OG1, Mustian KM, Peppone LJ, Janelsins M, Sprod LK, Kesler S et al. Impact of paroxetine on sleep problems in 426 cancer patients receiving chemotherapy: A trial from the University of Rochester Cancer Center Community Clinical Oncology Program. Sleep Med 2012;13:1184-90. Doi: 10.1016/j.sleep.2012.06.001

33. Musselman DL, Somerset WI, Guo Y, Manatunga AK, Porter $M$, Penna $S$, et al. A double-blind, multicenter, parallel-group study of paroxetine, desipramine, or pla- 
cebo in breast cancer patients (stages I, II, III, and IV) with major depression.. Clin Psychiatry 2006;67:288-296

34. Fisch MJ, Loehrer PJ, Kristeller J, Passik S, Jung $\mathrm{SH}$, Shen J, et al. Fluoxetine Versus Placebo in Advanced Cancer Outpatients: A Double-Blinded Trial of the Hoosier Oncology Group. J Clin Oncol 2003;21:193743. Doi: 10.1200/JCO.2003.08.025

35. Holland JC, Romano JC, Heiligenstein JH, Tepner RG, Wilson MG. A controlled trial of fluoxetine and desipramine in depressed women with advanced cancer. Psychooncology199;7:291-300. Doi:10.1002/ (SICI)1099-1611(199807/08)7:4<291::AIDPON361>3.0.CO;2-U

36. Li XJ, Dai ZY, Zhu BY, Zhen JP, Yang WF, Li DQ. Effects of sertraline on executive function and quality of life in patients with advanced cancer. Med Sc Monit 2014:20:1267-73. Doi: 10.12659/ MSM.890575.

37. Torta R, Siri I, Caldera P. Sertraline effectiveness and safety in depressed oncological patients. Support Care Cancer 2008;16:8391. Doi: 10.1007/s00520-007-0269-0

38. Ladd CO, Newport DJ, Ragan KA, Loughhead A, Stowe ZN. Venlafaxine in the treatment of depressive and vasomotor symptoms in women with perimenopausal depression. Depress Anxiety 2005;22:947. Doi:10.1002/da.20085

39. Boekhout AH1, Vincent AD, Dalesio OB, van den Bosch J, Foekema-Töns JH, Adriaansz S, et al. Management of hot flashes in patients who have breast cancer with venlafaxine and clonidine: A randomized, double-blind, placebo-controlled trial. J Clin Oncol 2011;29:3862-8. Doi: 10.1200/JCO.2010.33.1298.

40. Torta R1, Leombruni P, Borio R, Castelli L. Duloxetine for the treatment of mood disorder in cancer patients: a 12-week case-control clinical trial. Human psychopharmacol 2011;26:291-9. Doi: 10.1002/hup.1202

41. Theobald DE1, Kirsh KL, Holtsclaw E, Donaghy K, Passik SD. An open-label, cros- sover trial of mirtazapine (15 and $30 \mathrm{mg}$ ) in cancer patients with pain and other distressing symptoms. J Pain Symptom Manage 2002;23:442-7. Doi:10.1016/S08853924(02)00381-0

42. Ng CG1, Boks MP2, Roes KC3, Zainal NZ4, Sulaiman AH4, Tan SB5, et al. Rapid response to methylphenidate as an add-on therapy to mirtazapine in the treatment of major depressive disorder in terminally ill cancer patients: A fourweek, randomized, double-blinded, placebo-controlled study. Eur Neuropsychopharmacol 2014;24:491-8. Doi: 10.1016/j.euroneuro.2014.01.016

43. Mohammadinejad P, Arya P, Esfandbod M, Kaviani A, Najafi M, Kashani L. roIled Clinical Trial William CcCCCelecoxib versus diclofenac in mild to moderate depression management among breast cancer patients: A double-blind, placebo-controlled, randomized trial. Ann Pharmacother 2015:49:953-96. Doi: 10.1177/1060028015592215.

44. Hansen MV, Andersen LT, Madsen MT, Hageman I, Rasmussen LS, Bokmand S, et al. Effect of melatonin on depressive symptoms and anxiety in patients undergoing breast cancer surgery: a randomized, double-blind, placebo-controlled trial. Breast Cancer Res Treat 2014;145:683-95. Doi: 10.1007/s10549-014-2962-2

45. Rodin G, Lloyd N, Green E, Mackay JA, Wong RK. Treatment of depression in cancer patients". Curr Oncol 2007;14:180-7.

46. Capuron L1, Gumnick JF, Musselman $\mathrm{DL}$, Lawson DH, Reemsnyder A, Nemeroff $\mathrm{CB}$, et al. Neurobehavioral effects of interferon- ${ }^{a}$ in cancer patients: Phenomenology and paroxetine responsiveness of symptom dimensions. Neuropsychopharmacol 2002;26:643-52.

47. Morrow GR, Hickok JT, Roscoe JA, Raubertas RF, Andrews PLR, Flynn PJ. et al. Differential effects of paroxetine on fatigue and depression: a randomized, doubleblind trial from the University of Rochester 
Cancer Center Community Clinical Oncology Program. J Clin Oncol 2003;21:463541. DOi10.1200/JCO.2003.04.070

48. Barton DL, Loprinzi CL, Novotny P, Shanafelt T, Sloan J, Wahner-Roedler D, et al. Pilot evaluation of citalopram for the relief of hot flashes. J Support Oncol 2003;1:4751.

49. Theobald DE, Kirsh KL, Holtsclaw E, Donaghy K, Passik S.D. An open label pilot study of citalopram for depression and boredom in ambulatory cancer patients. Palliat Support Care 2003;1:71-7.

50. Grassi L, Biancosino B, Marnai L, Righi R. Effect or reboxetine on mayor depressive disorder in breast cancer patients: An open- label study. J Clin Psychiatry 2004;65:515-20.

51. Pae C-U, Kim Y-J, Won W-Y, Kim H-J, Lee $\mathrm{S}$, Lee $\mathrm{C}-\mathrm{U}$, et al. Paroxetine in the treatment of depressed patients with haematological malignancy: An open-label study. Hum Psychopharmacol Clin Exp 2004, 19: 25-29. Doi: 10.1002/hup.541

52. Gothelf D, Rubinstein M, Shemesh E, MiIler O, Farbstein I, Klein A. Pilot study: Fluvoxamine treatment for depression and anxiety disorders in children and adolescents with cancer. J Am Acad Child Adolesc Psychiatry 2005;44:1258-62.

53. Moss EL, Simpson JSA, Pelletier G, Forsyth P. An open-label study of the effects of bupropion sr on fatigue, depression and quality of life of mixed-site cancer patients and their partners. Psychoncology 2006;15:259-67. Doi:10.1002/pon.952

54. Stockler M.R, O'Connell R, Nowak A.K., Goldstein D, Turner J, Wilcken NRC. Effect of sertraline on symptoms and survival in patients with advanced cancer, but without major depression: a placebo-controlled double-blind randomised trial. Lancet Oncol 2007; 8:603-12. Doi: 10.1016/ S1470-2045(07)70148-1

55. Kim S-W, Shin I-S, Kim J-M, Kim Y-C, Kim $\mathrm{K}-\mathrm{S}, \mathrm{Kim} \mathrm{K}-\mathrm{M}$ et al. Effectiveness of mirtazapine for nausea and insomnia in cancer patients with depression. Psychiatry Clin Neurosci 2008;62:75-83. Doi:10.1111/ j.1440-1819.2007.01778.x

56. Lydiatt WM1, Denman D, McNeilly DP, Puumula SE, Burke WJ. Randomized, placebo-controlled trial of citalopram for the prevention of major depression during treatment for head and neck cancer. Arch Otolaryngol Head Neck Surg 2008;134:528-35. Doi: 10.1001/archotol.134.5.528.

57. Rodríguez Vega B, Palao A, Torres G, Benito G, E. Pérez E, Dieguez M. et al. Combined therapy versus usual care for the treatment of depression in oncologic patients: A randomized controlled trial. Psychooncology 2011;20:943-52. Doi: 10.1002/pon. 1800

58. Suzuki N, Ninomiya M, Maruta T, Hosonuma S, Yoshioka N, Ohara T, et al. Clinical study on the efficacy of fluvoxamine for psychological distress in gynecologic cancer patients. Int J Gynecol Cancer 2011;21:1143-9. Doi: 10.1097/ IGC.0b013e3181ffbeb9

59. Amodeo L, Castelli L, Leombruni P, Cipriani D, Biancofiore A, Torta R. Slow versus standard up-titration of paroxetine for the treatment of depression in cancer patients: A pilot study. Support Care Cancer 2011;20:375-84. Doi: 10.1007/s00520011-1118-8

60. Rodríguez Vega B, Orgaz Barnier P, Bayón C, Palao Á, Torres G, Hospital A, et al. Differences in depressed oncologic patients' narratives after receiving two different therapeutic interventions for depression: A qualitative study. Psychooncology 2012;21:1292-8. Doi: 10.1002/ pon.2036.

61. Park EM, Raddin RS, Nelson KM, Hamer RM, Mayer DK, Bernard SA et al. Conducting an antidepressant clinical trial in oncology: challenges and strategies to address them. Gen Hosp Psychiatry, 2012;36:474-6. Doi: 10.1016/j.genhosppsych.2014.05.007 
62. Nuñez GR, Pinczowski $H$, Zanellato R, Tateyama L, Schindler F, Fonseca F, et al. Bupropion for control of hot flashes in breast cancer survivors: a prospective, double-blind, randomized, crossover, pilot phase II trial. J Pain Symptom Manage 2013;45:969-79. Doi: 10.1016/j.jpainsymman.2012.06.011

63. Musselman D, Royster EB, Wang M, Long Q, Trimble LM, Mann TK, et al. The impact of escitalopram on il-2-induced neuroendocrine, immune, and behavioral changes in patients with malignant melanoma: preliminary findings. Neuropsychopharmacol 2013;38:1921-8. Doi: 10.1038/ npp.2013.85.
64. Lydiatt M, Bessette Diane K, Schmid Kendra, Sayles Harlan, Burke William J. Prevention of depression with escitalopram in patients undergoing treatment for head and neck cancer randomized, double-blind, placebo-controlled clinical trial. JAMA Otolaryngol Head Neck Surg 2013;139:67886. Doi: 10.1001/jamaoto.2013.3371.

65. Hansen MV, Andersen LT, Madsen MT, Hageman I, Rasmussen LS., Bokmand S, et al. Effect of melatonin on depressive symptoms and anxiety in patients undergoing breast cancer surgery: a randomized, double-blind, placebo-controlled trial. Breast Cancer Res Treat 2014;145:683-95. Doi: 10.1007/s10549-014-2962-2 